\title{
Large Right Ventricle Thrombus in Uhl's Anomaly: A Rare Presentation of Extremely Rare Disease
}

\author{
Gurkirat Singh ${ }^{\text {a, b }}$, Hemant Khemani ${ }^{\text {a }}$, Shakil Shaikha ${ }^{\mathrm{a}}$, Arun Bade ${ }^{\mathrm{a}}$, \\ Rahul Singla ${ }^{\mathrm{a}}$, Narender Omprakash Bansal ${ }^{\mathrm{a}}$
}

\begin{abstract}
Uhl's anomaly is an extremely rare congenital cardiac malformation and is characterized by the partial or complete absence of right ventricular myocardium. The absence of myocardium may be the result of primary non-development of myocytes or a form of selective apoptosis. It is mainly sporadic although some familial occurrences have been reported. Congestive cardiac failure is the most common mode of presentation. Associated congenital cardiac malformations are also reported. We report a case of a 17-year-old male who presented with symptoms and signs of right heart failure, during evaluation found to have large right ventricle free wall thrombus.
\end{abstract}

Keywords: Right ventricle thrombus; Uhl's anomaly; Congestive cardiac failure

\section{Introduction}

Uhl's anomaly is an extremely rare congenital cardiac malformation characterized by the partial or complete absence of right ventricular myocardium. The endocardium and the epicardium become opposed, but the septal component, septomarginal trabeculation, and the papillary muscles of the tricuspid valve (TV) are normally muscularized. The absence of myocardium may be the result of primary non-development of myocytes or a form of selective apoptosis. It is mainly sporadic although some familial occurrences have been reported. William Osler in 1905 described the term "parchment heart" [1], but the first case was reported by Henry Uhl in 1952 [2]. Gerlis et al in 1993 reported only 84 cases since the beginning of the 20th century [3]. Here we report a case of a 17-year-old male who presented with symptoms of right heart failure and was subsequently also diagnosed to have large right ventricle

Manuscript submitted July 9, 2018, accepted July 4, 2018

a Department of Cardiology, Grant Medical College and Sir J.J. Group of Hospitals, Mumbai, India

${ }^{\mathrm{b}}$ Corresponding Author: Gurkirat Singh, Department of Cardiology, Fourth Floor, Main Hospital Building, J.J. Hospital Campus, Byculla, Mumbai-400008, India. Email: singhgs269@yahoo.com

doi: https://doi.org/10.14740/cr758w thrombus.

\section{Case Report}

A 17-year-old male presented with gradually progressive bilateral pedal edema since 2 months. He also had gradually progressive distension of abdomen since 1 month. On examination, pulse was 84 per minute, irregularly irregular. Blood pressure was 106/68 mm Hg. There was bilateral pedal edema extending up to middle third of shin, moderate ascites and one finger hepatomegaly below right costal margin. Jugular venous pressure (JVP) was not in proportion with pedal edema and ascites, non pulsatile, and no cyanosis. Median sternotomy scar was present. Cardiac examination revealed cardiomegaly, diffuse apical impulse. The respiratory examination was normal. Parents gave a history of heart surgery under general anesthesia at the age of 2 years old when he was admitted with heart failure. After the procedure, he was discharged in 2 weeks and was asymptomatic. There was no history of cyanosis in past. They lost to follow-up after the procedure.

On routine investigations, hemoglobin was $9.7 \mathrm{~g} / \mathrm{dL}$, total leukocyte count $9,700 / \mu \mathrm{L}$ (neutrophils $68 \%$, lymphocytes $28 \%$, monocytes $4 \%$ ), and platelet count $120,000 / \mu \mathrm{L}$, bilirubin $2.2 \mathrm{mg} / \mathrm{dL}$ (direct fraction $1.1 \mathrm{mg} / \mathrm{dL}$ ), aspartate aminotransferase $124 \mathrm{U} / \mathrm{L}$, alanine aminotransferase $168 \mathrm{U} / \mathrm{L}$, serum albumin $3.3 \mathrm{~g} / \mathrm{dL}$, and serum globulin $3.9 \mathrm{~g} / \mathrm{dL}$; serum urea 44 $\mathrm{mg} / \mathrm{dL}$ creatinine $1.5 \mathrm{mg} / \mathrm{dL}$. Hepatitis B surface antigen, hepatitis $\mathrm{C}$ antibody, and human immunodeficiency virus (HIV) antibodies were negative.

Electrocardiogram (ECG) showed atrial fibrillation with controlled ventricular rate, diminished QRS complexes (Fig. 1). Chest X-ray showed situs solitus, levocardia and cardiomegaly (Fig. 2). Echocardiography showed gross dilatation of the right atrium and ventricle, severely depressed right ventricle (RV) function with good left ventricle (LV) function (Fig. 3a, b). A large thrombus was seen in RV, attached to the free wall in multiple views (Fig. 4a, b). Inferior vena cava (IVC) was dilated to $28 \mathrm{~mm}$ with minimal respiratory variations. The interatrial and interventricular septum was intact. RV outflow tract was free from thrombus. Patent bidirectional cavopulmonary anastomosis was seen in suprasternal view (Fig. 5). Subcostal saggital views and coronal view were not possible due to narrow subcostal angle. Cardiac computerized tomography (CT) was done in view of time 


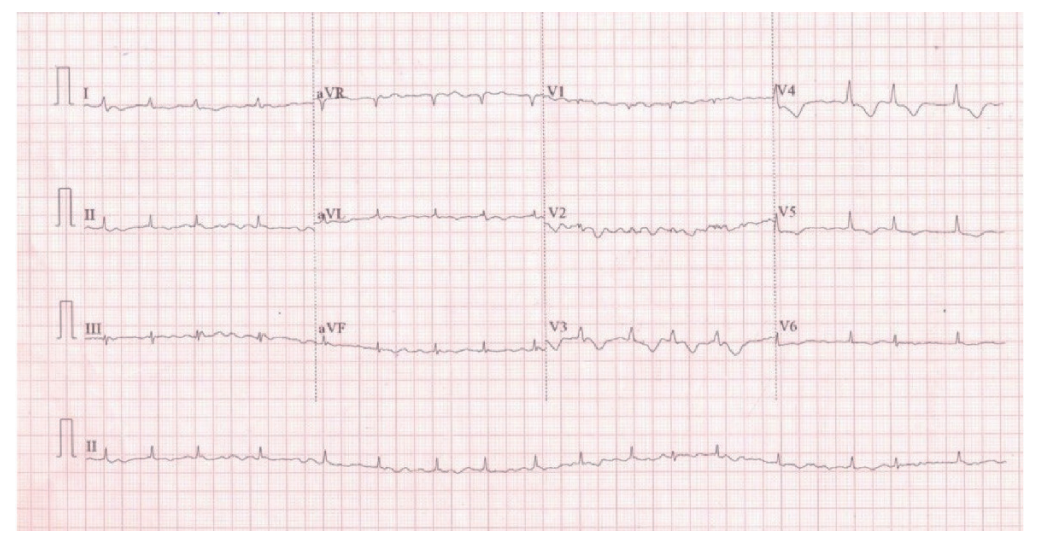

Figure 1. ECG showed atrial fibrillation and controlled ventricular rate.

constraints and unavailability of cardiac magnetic resonance imaging (MRI). Cardiac CT showed grossly dilated right atrium (RA) and RV (Fig. 6). Large RV free wall thrombus was seen measuring $10 \times 4.5 \mathrm{~mm}$ (Fig. 6). Superior vena cava (SVC) to right pulmonary artery shunt was patent. The interatrial septum was intact.

He was treated with anti-failure medications. Pedal edema and ascites reduced, and he was started on injection of unfractionated heparin as per weight 6 hourly for thrombus. As per the decision of our heart team, he was taken for excision of RV thrombus in view of the risk of life-threatening pulmonary thromboembolism. RV thrombus was excised through right atrial approach, Midline sternotomy. The right ventricle was markedly dilated, and its free wall was extremely thin and hardly contracting. Patient was stable post procedure and discharged on 10th day of procedure on oral medications including diuretics, beta-blocker, and warfarin 5 $\mathrm{mg}$. The importance of regular follow-up was explained. The need for cardiac transplantation in the future was explained

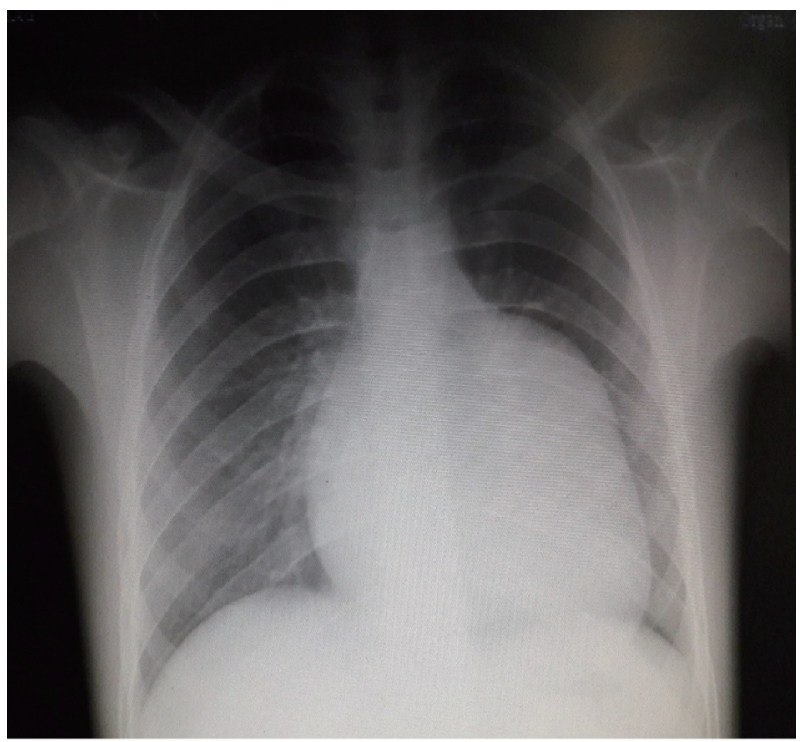

Figure 2. Chest X-ray showed cardiomegaly with the normal pulmonary flow. to the parents.

\section{Discussion}

Uhl's anomaly is an extremely rare form of congenital heart disease characterized by a partial or complete absence of the right ventricular myocardium, and preserved septal and left ventricular myocardium. It was probably first described by Osler as "parchment heart" in 1905 [1]. The anomaly is named after Henry Uhl, who reported the first case in 1952 [2]. Gerlis et al in 1993 reported only 84 cases since the beginning of the 20 th century [3]. It is mainly sporadic although some familial occurrences have been reported.

The pathogenesis of this anomaly is still unclear, but different theories have been postulated. The absence of myocardium may be the result of primary non-development of myocytes or a form of selective apoptosis [4]. Feucht et al [5] suggested the role of vascular endothelial growth factor in the induction of this cardiovascular malformation. The over expression of vascular endothelial growth factor, possibly by myocytes, may lead to impaired development of myocardium. It can be rarely associated with other congenital cardiac malformations like atrial septal defect (ASD), pulmonary atresia with an intact ventricular septum. Most cases end fatally in infancy or childhood, but rare adult cases have been reported. Traditionally, the diagnosis is made postmortem on autopsy specimens. However, with advances in medical technology, diagnosis can be made clinically and on imaging studies, most commonly echocardiography and cardiac MRI $[6,7]$.

Most common presentation is congestive cardiac failure. Arrhythmias and sudden cardiac death may also occur [8]. Medical treatment is only supportive. Early diagnosis and surgical intervention can prevent mortality. Surgical options include right ventricular exclusion: with atrial septectomy, bidirectional cavopulmonary anastomosis, and closure of the TV orifice [9], one and half ventricle repair with bidirectional cavopulmonary anastomosis and partial right ventriculectomy and atrial septectomy $[10,11]$, and ultimately cardiac transplantation. But surgical interventions failed to prolong life. Complications like arrhythmia, thrombosis, right ventricu- 

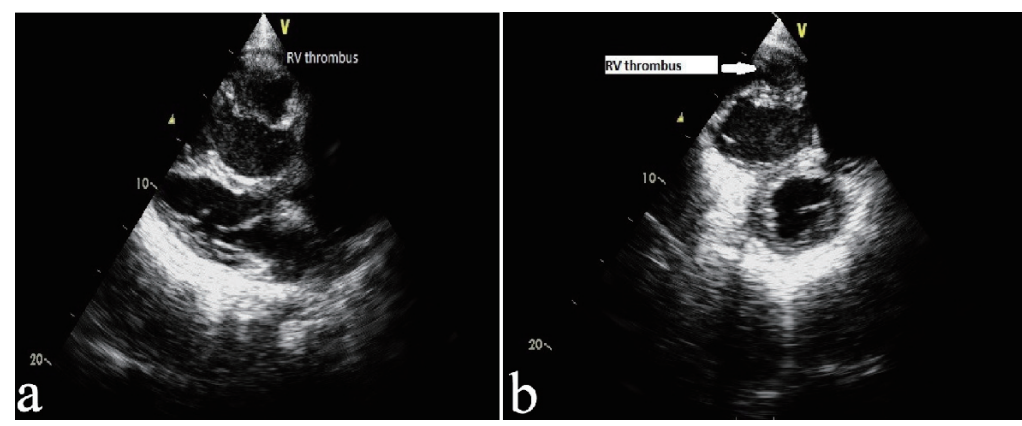

Figure 3. (a) Echocardiography, parasternal long axis view showing dilated RV and thrombus attached to RV free wall. (b) Echocardiography, parasternal short axis view showing dilated RV with large thrombus attached to free wall.
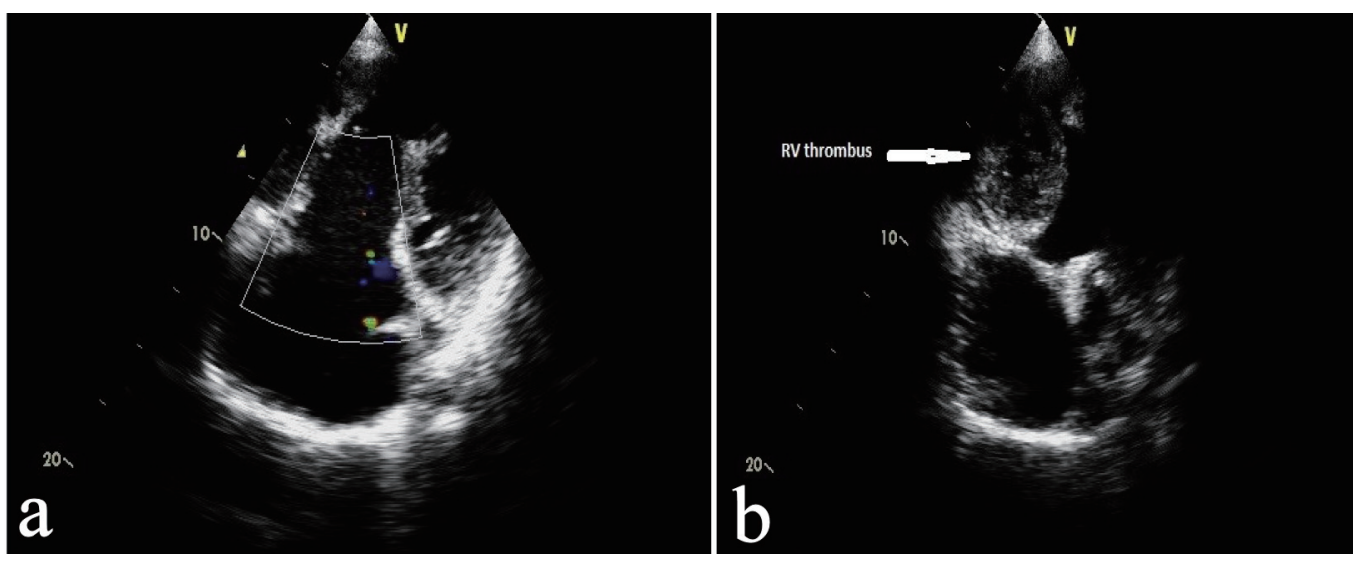

Figure 4. (a) Echocardiography, apical 4-chamber view, showing dilated RA, dilated RV, large RV free wall thrombus. (b) Echocardiography modification of apical 4-chamber view showed large RV free wall thrombus.

lar failure still occur in the life of post-surgical patients. In our case even after bidirectional cavopulmonary anastomosis, which was done at the age of 2 years, he developed large thrombus in RV which required early intervention to prevent life-threatening pulmonary embolism. He also had atrial fibrillation which was most probably chronic due to the large RA. It may also lead to thrombus formation in the left atrium and

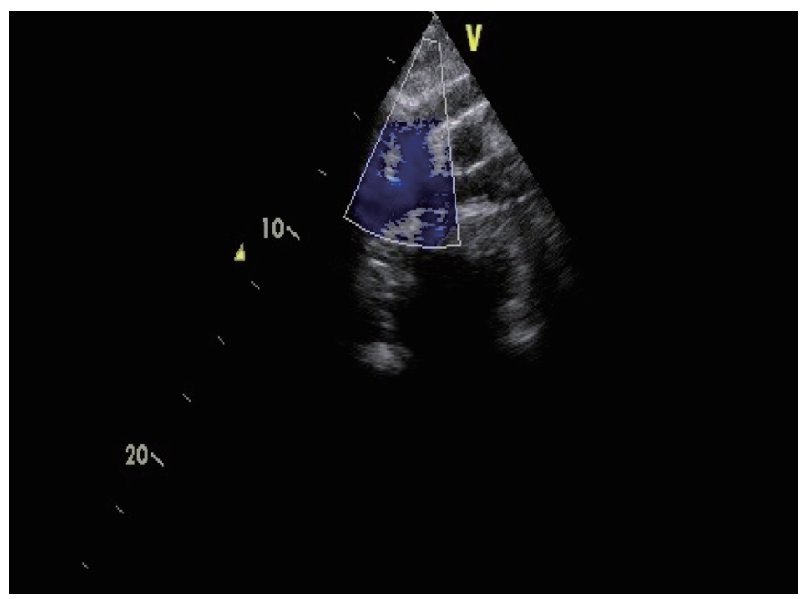

Figure 5. Echocardiography, supra-sternal view showing patent bidirectional cavopulmonary anastomosis. subsequently systemic embolism.

\section{Conclusions}

Uhl's anomaly is an extremely rare form of congenital heart disease and most commonly present as congestive cardiac failure. Traditionally the diagnosis was made postpartum; but advances in medical technology have enabled us to diagnose this

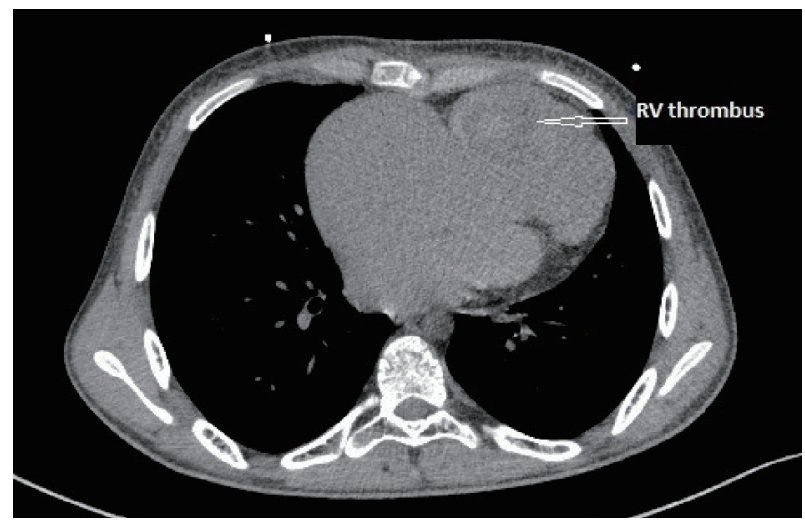

Figure 6. Cardiac CT axial view showing grossly dilated right atrium, right ventricle and large RV free wall thrombus with thin RV free wall. 
anomaly early. Early diagnosis and surgical intervention can prevent mortality, but as the age advances other complications like arrhythmia and thrombosis lead to significant mortality and morbidity. Even after surgical intervention life expectancy is not normal.

\section{References}

1. Osler WM. The Principles and Practice of Medicine. 6th ed. New York: D. Appleton; 1905. p. 280.

2. Uhl HS. A previously undescribed congenital malformation of the heart: almost total absence of the myocardium of the right ventricle. Bull Johns Hopkins Hosp. 1952;91(3):197-209.

3. Gerlis LM, Schmidt-Ott SC, Ho SY, Anderson RH. Dysplastic conditions of the right ventricular myocardium: Uhl's anomaly vs arrhythmogenic right ventricular dysplasia. Br Heart J. 1993;69(2):142-150.

4. Gould L, Guttman AB, Carrasco J, Lyon AF. Partial absence of the right ventricular musculature. A congenital lesion. Am J Med. 1967;42(4):636-641.
5. Feucht M, Christ B, Wilting J. VEGF induces cardiovascular malformation and embryonic lethality. Am J Pathol. 1997;151(5):1407-1416.

6. Cheng JF, Mohammed TL, Griffith BP, White CS. CT of Uhl's anomaly in an adult. Int J Cardiovasc Imaging. 2005;21(6):663-666.

7. Greer ML, MacDonald C, Adatia I. MRI of Uhl's anomaly. Circulation. 2000;101(24):E230-232.

8. Song BG. A rare case of partial absence of the right ventricular musculature in asymptomatic adult man: partial Uhl's anomaly. Heart Lung. 2013;42(3):215-217.

9. Azhari N, Assaqqat M, Bulbul Z. Successful surgical repair of Uhl's anomaly. Cardiol Young. 2002;12(2):192-195.

10. Yoshii S, Suzuki S, Hosaka S, Osawa H, Takahashi W, Takizawa K, Abraham SJ, et al. A case of Uhl anomaly treated with one and a half ventricle repair combined with partial right ventriculectomy in infancy. J Thorac Cardiovasc Surg. 2001;122(5):1026-1028.

11. Ikari NM, Azeka E, Aiello VD, Atik E, Barbero-Marcial M, Ebaid M. Uhl's anomaly. Differential diagnosis and indication for cardiac transplantation in an infant. Arq Bras Cardiol. 2001;77(1):69-76. 